\title{
Tandem High-Dose Chemotherapy and Autologous Stem Cell Transplantation for Atypical Teratoid/Rhabdoid Tumor
}

Ki Woong Sung, MD, PhD'1
Do Hoon Lim, MD, PhD²
Eun Sang Yi, MD'
Young Bae Choi, MD
Ji Won Lee, MD, PhD'
Keon Hee Yoo, MD, PhD
Hong Hoe Koo, MD, PhD'
Ji Hye Kim, MD, PhD
Yeon-Lim Suh, MD, PhD
Yoo Sook Joung, MD, PhD'
Hyung Jin Shin, MD, PhD

Departments of ${ }^{1}$ Pediatrics, ${ }^{2}$ Radiation Oncology, ${ }^{3}$ Radiology, ${ }^{4}$ Pathology, ${ }^{5}$ Psychiatry, and ${ }^{6}$ Neurosurgery, Samsung Medical Center, Sungkyunkwan University School of Medicine, Seoul, Korea

Correspondence: Hyung Jin Shin, MD, PhD Department of Neurosurgery,

Samsung Medical Center, Sungkyunkwan

University School of Medicine, 81 Irwon-ro, Gangnam-gu, Seoul 06351, Korea

Tel: 82-2-3410-3492

Fax: 82-2-3410-0043

E-mail: shinhj@skku.edu

Received September 10, 2015

Accepted March 23, 2016

Published Online April 1, 2016

*Ki Woong Sung and Do Hoon Lim contributed equally to this work.

\begin{abstract}
Purpose
We prospectively evaluated the effectiveness of tandem high-dose chemotherapy and autologous stem cell transplantation (HDCT/auto-SCT) in improving the survival of patients with atypical teratoid/rhabdoid tumors while reducing the risks of late adverse effects from radiotherapy (RT).
\end{abstract}

\section{Materials and Methods}

For young children ( $<3$ years old), tandem HDCT/auto-SCT was administered after six cycles of induction chemotherapy. RT was deferred until after 3 years of age unless the tumor showed relapse or progression. For older patients ( $>3$ years old), RT including reduceddose craniospinal RT ( 23.4 or 30.6 Gy) was administered either after two cycles of induction chemotherapy or after surgery, and tandem HDCT/auto-SCT was administered after six cycles of induction chemotherapy.

\section{Results}

A total of 13 patients (five young and eight older) were enrolled from November 2004 to June 2012. Eight patients, including all five young patients, had metastatic disease at diagnosis. Six patients (four young and two older) experienced progression before initiation of $\mathrm{RT}$, and seven were able to proceed to HDCT/auto-SCT without progression during induction treatment. Three of six patients who experienced progression during induction treatment underwent HDCT/auto-SCT as salvage treatment. All five young patients died from disease progression. However, four of the eight older patients remain progression-free with a median follow-up period of 64 months (range, 39 to 108 months). Treatment-related late toxicities were acceptable.

\section{Conclusion}

The required dose of craniospinal RT might be reduced in older patients if the intensity of chemotherapy is increased. However, early administration of RT should be considered to prevent early progression in young patients.

\section{Introduction}

Central nervous system (CNS) atypical teratoid / rhabdoid tumors (ATRTs) are highly malignant. ATRT accounts for $1 \%-2 \%$ of CNS tumors in children of all ages, but 10\%-20\% in patients younger than 3 years $[1,2]$. ATRTs are distinguished from other embryonal brain tumors not only by the

\section{Key words}

Brain neoplasms, Chemotherapy,

Hematopoietic stem cell transplantation, Radiotherapy presence of rhabdoid cells and specific immunohistochemistry but also by biological markers [3]. CNS ATRTs are associated with significantly worse overall survival (OS) than other embryonal tumors [4-6]. The very poor prognosis of ATRT in young children may be related to the limited use of radiotherapy (RT) because of the risks of functional impairment of the developing brain and late adverse effects [7]. Multiple chemotherapeutic approaches have been attempted 
to defer radiation; however, OS remains dismal. For older children, a few studies have suggested that outcome might be improved with standard-dose craniospinal RT (CSRT) and high-dose alkylator-based chemotherapy [2]. However, various late adverse effects primarily attributable to RT, particularly CSRT, were unavoidable.

A treatment strategy using high-dose chemotherapy and autologous stem cell transplantation (HDCT/auto-SCT) has shown clinical benefit in children with high-risk or recurrent solid tumors including brain tumors [8-12], and recent studies have suggested that further dose intensification using tandem HDCT/auto-SCT might improve outcomes and/or reduce RT dose without jeopardizing survival rates [13-15]. Gajjar et al. [13] reported the results of a prospective study (SJMB 96 study) employing tandem HDCT/auto-SCT in patients with high-risk medulloblastoma. In their study, patients received 36.0-39.6 Gy of CSRT followed by four cycles of HDCT / auto-SCT, and the 5-year event-free survival (EFS) rate was 70\% [13]. Previous reports from our institution also suggested that tandem HDCT/auto-SCT with deferred and/or reduced RT was feasible and might further improve outcomes in young children with brain tumors and patients (>3 years of age) with high-risk medulloblastoma [13,14]. In the current study, we prospectively evaluated the effectiveness of tandem HDCT/auto-SCT in children with ATRT. The aim of our new treatment strategy was to improve patient survival while reducing the risks of late adverse effects from RT.

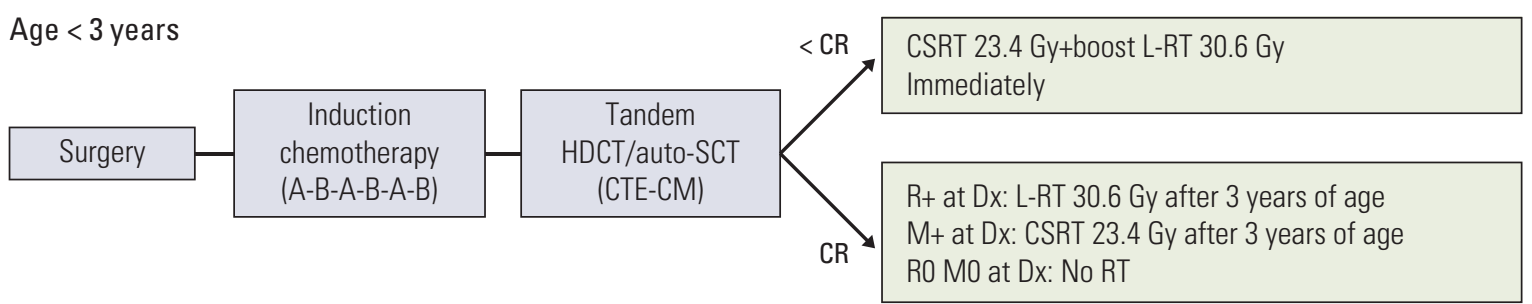

Age $>3$ years, until 2011

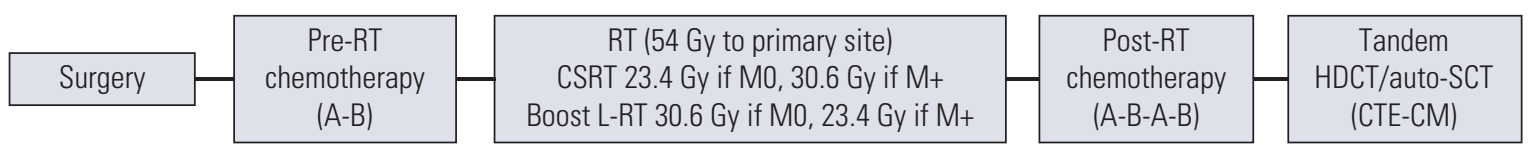

Age $>3$ years, from 2012

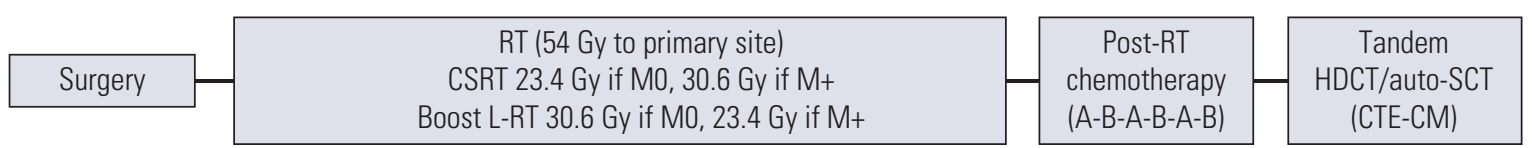

Fig. 1. Treatment scheme. For young children, six cycles of induction chemotherapy were administered prior to HDCT/autoSCT, consisting of alternating CECV and CEIV regimens. RT was either not administered or was deferred until after 3 years of age if the patient achieved complete response after tandem HDCT/ auto-SCT. Use of RT was determined according to the tumor status at the time of diagnosis. For older children, two cycles of pre-RT and four cycles of post-RT chemotherapy were administered during the early study period. During the late study period when RT was administered after surgery, six cycles of post-RT chemotherapy were administered. The regimens for the first and second HDCT/auto-SCT were CTE and CM, respectively. HDCT/ auto-SCT, high-dose chemotherapy and autologous stem cell transplantation; CECV, carboplatin+etoposide+cyclophosphamide+vincristine; CEIV, carboplatin+etoposide+ifosfamide+vincristine; RT, radiotherapy; CSRT, craniospinal RT; L-RT, local RT; Dx, diagnosis; CTE, carboplatin+thiotepa+etoposide; CM, cyclophosphamide+melphalan. 
Table 1. Chemotherapy regimens

\begin{tabular}{|c|c|c|c|c|}
\hline Regimen & Drug & Dose (mg/m²/day) & Schedule & Total dose $\left(\mathrm{mg} / \mathrm{m}^{2}\right)$ \\
\hline \multicolumn{5}{|c|}{ Induction regimen } \\
\hline \multirow[t]{4}{*}{$\mathrm{CECV}^{\mathrm{a})}$} & Cisplatin & 90 & Day 0 & 90 \\
\hline & Etoposide & 75 & Days 0-2 & 225 \\
\hline & Cyclophosphamide & 1,500 & Days 1 and 2 & 3,000 \\
\hline & Vincristine & 1.5 & Days 0 and 7 & 3.0 \\
\hline \multirow[t]{4}{*}{ (EIV ${ }^{\text {a) }}$} & Carboplatin & 300 & Days 0 and 1 & 600 \\
\hline & Etoposide & 75 & Days $0-4$ & 375 \\
\hline & Ifosfamide & 1,500 & Days $0-4$ & 7,500 \\
\hline & Vincristine & 1.5 & Days 0 and 7 & 3.0 \\
\hline \multicolumn{5}{|c|}{ HDCT regimen } \\
\hline \multirow[t]{3}{*}{ First: CTE } & Carboplatin & 500 & Days $-8,-7,-6$ & 1,500 \\
\hline & Thiotepa & 300 & Days $-5,-4,-3$ & 900 \\
\hline & Etoposide & 250 & Days $-5,-4,-3$ & 750 \\
\hline \multirow[t]{2}{*}{ Second: CM } & Cyclophosphamide & 1,500 & Days $-8,-7,-6,-5$ & 6,000 \\
\hline & Melphalan & 60 & Days $-4,-3,-2$ & 180 \\
\hline
\end{tabular}

CECV, carboplatin+etoposide+cyclophosphamide+vincristine; CEIV, carboplatin+etoposide+ifosfamide+vincristine; HDCT, high-dose chemotherapy; CTE, carboplatin+thiotepa+etoposide; CM, cyclophosphamide+melphalan. ${ }^{\text {a }}$ Dose was determined based on body weight in children under 3 years of age.

\section{Materials and Methods}

This study is a pilot study including a small cohort of patients with ATRT in a single institution. The study was approved by the Institutional Review Board of Samsung Medical Center (2004-12-009 and 2011-06-081), Seoul, Korea, and conducted in accordance with the Declaration of Helsinki and the Good Clinical Practice guidelines. All parents and guardians gave their written informed consent before enrollment.

\section{Patients}

Children diagnosed with ATRT from November 2004 to June 2012 were enrolled in the study. All cases and loss of nuclear expression of INI1 was confirmed by immunohistochemical staining by a pediatric neuropathologist. Disease extent at diagnosis was assessed using brain and spinal magnetic resonance imaging (MRI) and cerebrospinal fluid (CSF) cytology.

\section{Induction chemotherapy}

The treatment scheme is shown in Fig. 1. Induction treatment was initiated within 4 weeks, and preferably within 3 weeks, after surgery. For young children, six cycles of induc- tion chemotherapy were administered prior to HDCT / autoSCT, and consisted of alternating CECV (cisplatin+etoposide +cyclophosphamide+vincristine) and CEIV (carboplatin+ etoposide+ifosfamide+vincristine) regimens (Table 1). RT was deferred until after 3 years of age unless the tumor showed relapse or progression. For older children, a total of six cycles of induction chemotherapy were administered prior to tandem HDCT / auto-SCT. During the early study period (diagnosis between November 2004 and December 2011), two cycles of pre-RT and four cycles of post-RT chemotherapy (75\% of calculated dose) were administered. During the late study period (diagnosis from January 2012), when RT was administered after surgery without preceding chemotherapy, six cycles of post-RT chemotherapy were administered. Post-RT chemotherapy was initiated within 4 weeks after completion of RT, and the doses were reduced by $25 \%$ of the calculated dose because of the long-lasting myelosuppressive effect of CSRT. Induction chemotherapy cycles were scheduled 28 days apart. If needed, delays were permitted to allow recovery of the absolute neutrophil count (ANC) and platelet count to 1,000 and $100,000 / \mathrm{mm}^{3}$, respectively. Peripheral blood stem cells (PBSCs) were collected during the recovery phase of the first chemotherapy cycle in the early study period, or after surgery (steady state mobilization with granulocyte-colony stimulating factor for 4-5 days) in the late study period. 


\section{Radiotherapy}

For young children, RT was either not given or was deferred until after 3 years of age if the patient achieved complete response (CR) after tandem HDCT/auto-SCT. Use of RT was determined according to the tumor status at the time of diagnosis. Local RT (L-RT) by 3-dimensional conformal RT (30.6 Gy, 1.8 Gy/fraction) was administered after 3 years of age only if gross residual tumor $\left(>1.5 \mathrm{~cm}^{2}\right)$ remained after initial or second-look surgery. For L-RT to the primary site, the radiation target volume was defined as the postoperative surgical cavity and residual tumor (if present) with 1-2 cm margins. CSRT (23.4 Gy, $1.8 \mathrm{~Gy} /$ fraction) was administered after 3 years of age only if leptomeningeal seeding was present at the time of diagnosis. However, both CSRT and boost L-RT were administered immediately if the patient had not achieved CR after tandem HDCT / auto-SCT (Fig. 1).

For older children with primary tumor at cerebrum, during the early study period, RT consisted of 23.4 Gy (30.6 Gy if M+) of CSRT, 30.6 Gy (23.4 Gy if M+) of boost L-RT to the primary site, and $14.4 \mathrm{~Gy}$ of boost RT to the gross seeding nodule (if present). During the early study period, two patients experienced progression before initiation of RT. Therefore, in the late study period, RT was administered after surgery without preceding chemotherapy.

\section{Tandem HDCT/auto-SCT}

The regimens for the first and second HDCT/auto-SCT were CTE (carboplatin+thiotepa+etoposide) and CM (cyclophosphamide+melphalan), respectively (Table 1). An approximately 12-week interval without treatment was allowed between the first and second HDCT/auto-SCT. Approximately half of the PBSCs collected during a single round of leukapheresis were infused for marrow rescue during each HDCT/auto-SCT session.

\section{Response and toxicity criteria}

Disease response was evaluated by brain and spinal MRI and CSF cytology every two cycles of chemotherapy, after RT, between the first and second HDCT / auto-SCT, every 3 months for the first year after completion of HDCT/autoSCT, every 4 months for the second year, and every 6 months thereafter. Tumor size was estimated by MRI as the product of the greatest diameter and the longest perpendicular diameter. Disease responses were categorized as follows: progressive disease, greater than a $25 \%$ increase in tumor size or the appearance of a new area of tumor; stable disease, less than a $25 \%$ change in tumor size; minor response, $25 \%$ to $50 \%$ decrease in tumor size; partial response, greater than a 50\% decrease in tumor size; $\mathrm{CR}$, complete disappearance of all previously measurable tumor; or continuous $\mathrm{CR}$, continuous $\mathrm{CR}$. Toxicities were graded using the Common Terminology Criteria ver. 4.0 of the National Cancer Institute.

\section{Evaluation of late adverse effects}

Late adverse effects were evaluated annually after completion of HDCT/ auto-SCT. The diagnosis of growth hormone deficiency was based on a declining growth rate and was confirmed by biochemical testing. Hypothyroidism was diagnosed by elevation of thyrotropin. Adrenal insufficiency was diagnosed based on the failure to increase cortisol levels after administration of corticotropin releasing hormone. Cognitive function was evaluated using the Korean-Wechsler Intelligence Scale for Children-IV or Korean-Wechsler Adult Intelligence Scale-IV. Cardiac, renal, hepatic, auditory, and ophthalmologic functions were also evaluated.

\section{Statistics}

This study is a pilot study including a small cohort of patients with ATRT. The primary aim of the study is to improve patient survival while reducing the risks of late adverse effects from RT. EFS was calculated from the date of diagnosis until the date of relapse, progression, or death, whichever occurred first. OS was calculated from the date of diagnosis until death from any cause. Survival rates and standard errors were estimated using the Kaplan-Meier method, and differences in survival rates between the two groups were compared using the log-rank test. Differences in the frequencies of clinical characteristics between the two age groups were analyzed using a chi-square test or Fisher exact test. $p$-values less than 0.05 were considered significant.

\section{Results}

\section{Patient characteristics}

Thirteen consecutive patients (three boys and 10 girls) with ATRT were enrolled during the study period. Patient characteristics are listed in Table 2. The median age at diagnosis was 79 months (range, 2 to 181 months), and five patients were younger than 3 years at diagnosis. Gross total resection or near total resection (>90\% resection) was possible in five patients and subtotal resection (50\%-90\% resection) or less was performed in the eight remaining patients. Eight patients had metastatic disease (M1 in one, M2 in one, and M3 in six according to the Chang staging system [16]). Four patients had tumors at the posterior fossa, eight at the supra- 
Table 2. Grade 3 / 4 toxicity profile of induction chemotherapy

\begin{tabular}{|c|c|c|c|c|c|}
\hline \multirow{2}{*}{ Parameter } & \multicolumn{2}{|c|}{ Pre-RT chemotherapy } & \multicolumn{2}{|c|}{ Post-RT chemotherapy } & \multirow{2}{*}{$\begin{array}{c}\text { Total } \\
\text { (62 cycles) }\end{array}$} \\
\hline & CECV (18 cycles) & CEIV (17 cycles) & CECV (14 cycles) & CEIV (13 cycles) & \\
\hline \multicolumn{6}{|l|}{ Hematologic toxicity } \\
\hline Chemotherapy dose $(\%)^{\mathrm{a})}$ & $99.6(79.5-104.5)$ & $100(79.1-102.8)$ & $74.5(70.4-76.7)$ & $73.6(69.5-76.5)$ & - \\
\hline Dose reduction $>5 \%$ & $1(5.6)$ & $1(5.9)$ & 0 & $1(7.7)$ & $3(4.8)$ \\
\hline Interval to next cycle (day) & $29(24-58)$ & $32(25-37)$ & $28(26-39)$ & $32(27-38)$ & $29(24-58)$ \\
\hline Interval $>35$ days & $2(11.1)$ & $1(5.9)$ & $1(7.1)$ & $4(30.8)$ & $8(12.9)$ \\
\hline Delayed hematologic recovery & 0 & $1(5.9)$ & $1(7.1)$ & $2(15.4)$ & $4(6.5)$ \\
\hline Other causes & $2(11.1)^{b)}$ & 0 & 0 & $2(15.4)^{c}$ & $4(6.5)$ \\
\hline Duration of neutropenia (day) & $8(4-11)$ & $8(4-20)$ & $8(5-15)$ & $6(1-12)$ & $7.5(1-20)$ \\
\hline No. of platelet transfusions & $4(1-10)$ & $3(0-14)$ & $2(1-8)$ & $3(1-8)$ & $3(0-14)$ \\
\hline Neutropenic fever & $12(66.7)$ & $8(47.1)$ & $9(64.3)$ & $7(53.8)$ & $36(58.1)$ \\
\hline Positive blood culture & $1(5.6)$ & $2(11.8)$ & 0 & $1(7.7)$ & $4(6.5)$ \\
\hline \multicolumn{6}{|l|}{ Non-hematologic toxicity } \\
\hline Elevation of liver enzymes & $1(5.6)$ & $1(5.9)$ & 0 & 0 & $2(3.2)$ \\
\hline Hyperbilirubinemia & 0 & 0 & 0 & 0 & 0 \\
\hline Renal insufficiency & 0 & 0 & 0 & 0 & 0 \\
\hline Hypokalemia & $2(11.1)$ & $2(11.8)$ & 0 & $1(7.7)$ & $5(8.1)$ \\
\hline Hyponatremia & $1(5.6)$ & 0 & 0 & 0 & $1(1.6)$ \\
\hline
\end{tabular}

Values are presented as median (range) or number (\%). CECV, cisplatin+etoposide+cyclophosphamide+vincristine; CEIV, carboplatin+etoposide+ifosfamide+vincristine. a)Percent of calculated dose, ${ }^{\text {b}}$ Delay due to shunt problems, ${ }^{\text {c) Delay due to }}$ high-dose chemotherapy scheduling.

tentorial area, and one at the spinal cord. Although four of five young patients had tumors at the posterior fossa, all of the older patients had tumors at sites other than the posterior fossa ( $\mathrm{p}=0.010)$. All of the young patients had leptomeningeal seeding at diagnosis, compared with only three of eight older patients $(\mathrm{p}=0.024)$.

\section{Induction chemotherapy}

The grade $3 / 4$ toxicity profile during a total of 62 cycles of induction chemotherapy is shown in Table 2. Hematologic toxicity was generally acceptable. The median duration of neutropenia (ANC $<500 / \mathrm{mm}^{3}$ ) was 7.5 days (range, 1 to 20 days) and the median interval between chemotherapy cycles was 29 days (range, 24 to 58 days). Delay in scheduled chemotherapy of more than 7 days occurred in only eight cycles $(12.9 \%$ ) (four from delayed hematologic recovery, two from shunt problems, and two from HDCT scheduling), and chemotherapy dose reduction of more than $5 \%$ occurred in only three cycles $(4.8 \%)$. Neutropenic fever which was experienced by all patients occurred in 36 cycles $(58.1 \%)$ and four episodes $(6.5 \%)$ of blood stream infection were documented. Non-hematologic toxicity was not common during induction chemotherapy (Table 2). The response to induction treatment is shown in Table 3. Tumor relapse or progression during induction chemotherapy occurred in three of five young patients, two of whom died of tumor progression. Three of eight older patients experienced progression (two before RT and one after RT). Among these six cases of progression or relapse during induction treatment, four patients (one young and three older) received salvage treatment including RT and chemotherapy, and three (one young and two older) proceeded to HDCT/auto-SCT. During the three leukapheresis procedures performed per patient, the median number of CD $34^{+}$cells collected was $41.7 \times 10^{6} / \mathrm{kg}$ (range, $3.1 \times 10^{6} / \mathrm{kg}$ to $119.5 \times 10^{6} / \mathrm{kg}$ ).

\section{Radiotherapy}

The RT doses administered to 10 patients are shown in Table 3. Two of five young patients received 23.4 Gy of CSRT and 30.6 Gy of boost L-RT at 41 and 22 months of age, respectively, because they did not achieve CR after tandem HDCT/auto-SCT. The three remaining young patients did not receive RT due to death after progression or failure to obtain parental consent. Five older patients without leptomeningeal seeding also received 23.4 Gy of CSRT and 30.6 Gy of boost L-RT. One older M+ patient received 30.6 Gy of CSRT and 23.4 Gy of boost L-RT, and one patient (No. 11) received rescue RT (36.0 Gy of CSRT and 18.0 Gy of boost 


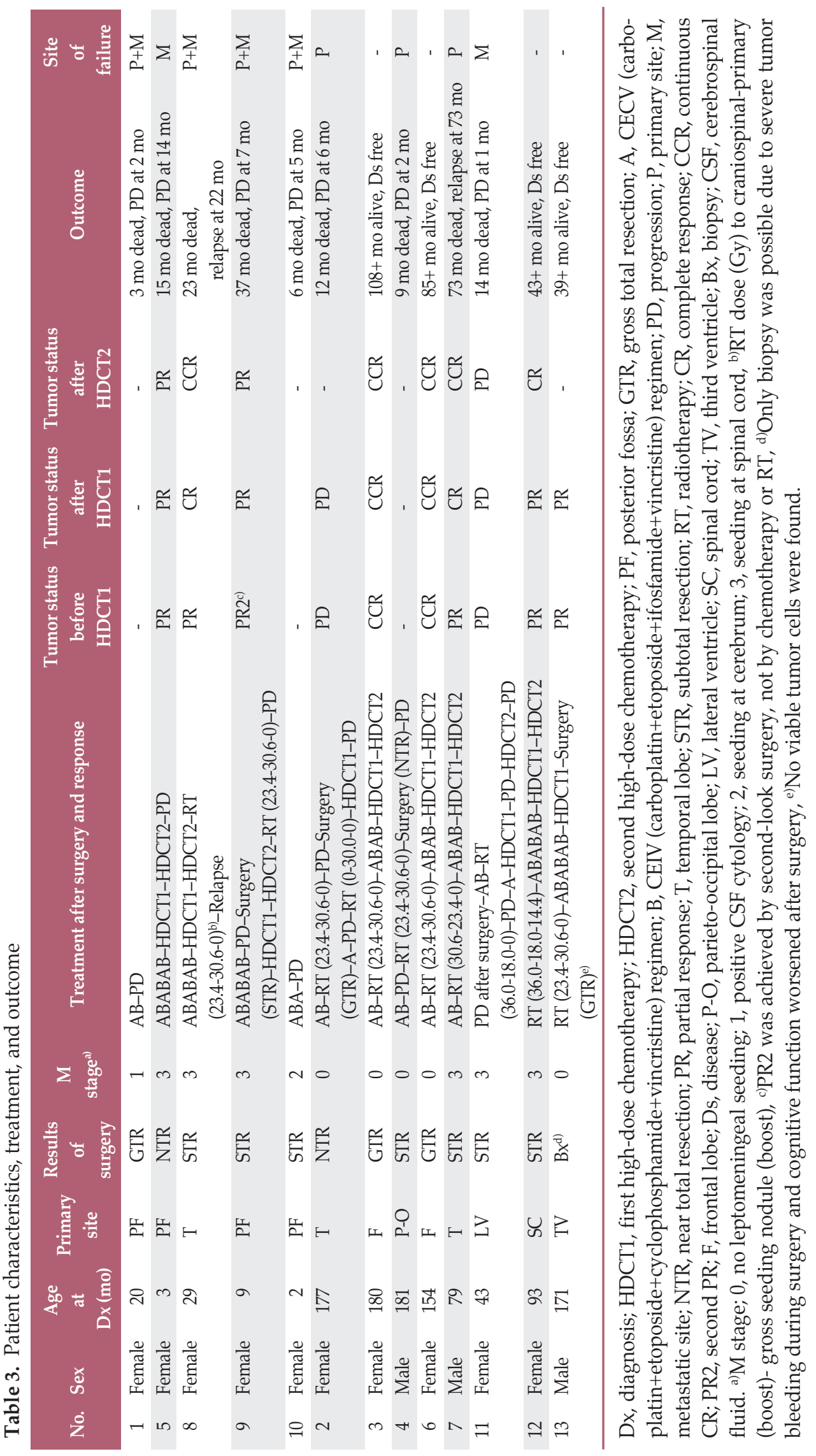


L-RT) after progression. The remaining patient (No. 12) with spinal ATRT received 36.0 Gy of CSRT, 18 Gy of boost L-RT to the primary site, and 14.4 Gy of boost RT to the seeding nodule. In summary, eight patients received RT as scheduled without deviation.

\section{Tandem HDCT/auto-SCT}

Seven patients could proceed to HDCT / auto-SCT without progression during induction treatment. Another three patients underwent HDCT / auto-SCT as salvage treatment after relapse/ progression during induction treatment. Eight of 10 patients who underwent the first HDCT/auto-SCT proceeded to the second HDCT/auto-SCT. The two remaining patients could not proceed to the second HDCT/autoSCT because of tumor progression (patient No. 2) and refusal by parent (patients No. 13), respectively. The median doses of HDCTs were $99.2 \%$ and $99.1 \%$ of the calculated dose based on body surface area in the first and second HDCT/autoSCTs, respectively. In one patient (patient No. 5) who was younger than 1 year at the time of HDCT / auto-SCT, the dose was calculated based on body weight. The median interval from day 0 of the first HDCT/auto-SCT to initiation of the second HDCT/ auto-SCT was 84 days (range, 75 to 93 days). The grade $3 / 4$ toxicity profile of patients receiving tandem HDCT/auto-SCT is shown in Table 4. Both the neutrophil and platelet counts recovered rapidly during the first and second HDCT / auto-SCT. During the first HDCT / auto-SCT, frequent grade $3 / 4$ toxicities included fever, stomatitis, elevation of liver enzymes, and hypokalemia. Toxicities were milder during the second HDCT/auto-SCT compared with the first. No treatment-related mortality (TRM) occurred during the tandem HDCT/ auto-SCT.

\section{Survival}

Tumor relapse or progression during induction treatment occurred in six (three young and three older) patients, all of whom died from tumor progression. All three patients who underwent HDCT/auto-SCT as salvage treatment after relapse/progression experienced progression again and died at 2, 2, and 25 months post-transplant. Among seven (two young and five older) patients who could proceed to HDCT/ auto-SCT without relapse or progression, three (two

Table 4. Grade 3 / 4 toxicity profile of tandem HDCT/ auto-SCT

\begin{tabular}{|c|c|c|c|}
\hline Parameter & First HDCT/auto-SCT (n=10) & Second HDCT/auto-SCT (n=8) & p-value \\
\hline \multicolumn{4}{|l|}{ Hematologic toxicity } \\
\hline $\mathrm{CD} 34^{+}$cells $\left(\times 10^{6} / \mathrm{kg}\right)$ & $10.3(1.7-47.5)$ & $14.0(1.3-40.2)$ & 0.633 \\
\hline Days $^{\text {a) }}$ to reach ANC $500 / \mathrm{mm}^{3}$ & $9(8-11)$ & $9.5(8-11)$ & 0.360 \\
\hline Days $^{\text {b) }}$ to reach PLT count $20,000 / \mathrm{mm}^{3}$ & $18(16-52)$ & $20(16-64)$ & 0.963 \\
\hline Days of $\mathrm{BT} \geq 38.0^{\circ} \mathrm{C}$ & $4.5(1-6)$ & $3(0-5)$ & 0.360 \\
\hline Positive blood culture (No. of patients) & 4 & 1 & 0.314 \\
\hline \multicolumn{4}{|l|}{ Non-hematologic toxicity (No. of patients) } \\
\hline Stomatitis & 7 & 1 & 0.025 \\
\hline Vomiting & 1 & 0 & 0.556 \\
\hline Diarrhea & 3 & 0 & 0.216 \\
\hline Elevation of liver enzymes & 7 & 0 & 0.004 \\
\hline Hyperbilirubinemia & 1 & 0 & 0.556 \\
\hline Renal insufficiency & 0 & 0 & $>0.999$ \\
\hline Hypokalemia & 5 & 1 & 0.036 \\
\hline Hyperkalemia & 0 & 0 & $>0.999$ \\
\hline Hyponatremia & 2 & 1 & 0.588 \\
\hline Hypernatremia & 0 & 0 & $>0.999$ \\
\hline Hepatic VOD & 1 & 1 & 0.706 \\
\hline Myocarditis & 0 & 0 & $>0.999$ \\
\hline Seizure & 2 & 0 & 0.477 \\
\hline Treatment-related mortality & 0 & 0 & $>0.999$ \\
\hline
\end{tabular}

Values are presented as median (range) or number. HDCT/auto-SCT, high-dose chemotherapy and autologous stem cell transplantation; ANC, absolute neutrophil count; PLT, platelet; BT, body temperature; VOD, veno-occlusive disease. ${ }^{\text {a) }}$ The first of 3 consecutive days that ANC exceeded $500 / \mathrm{mm}^{3}$, b) The first of 7 consecutive days that PLT count exceeded 20,000/ $\mathrm{mm}^{3}$ without transfusion. 
A

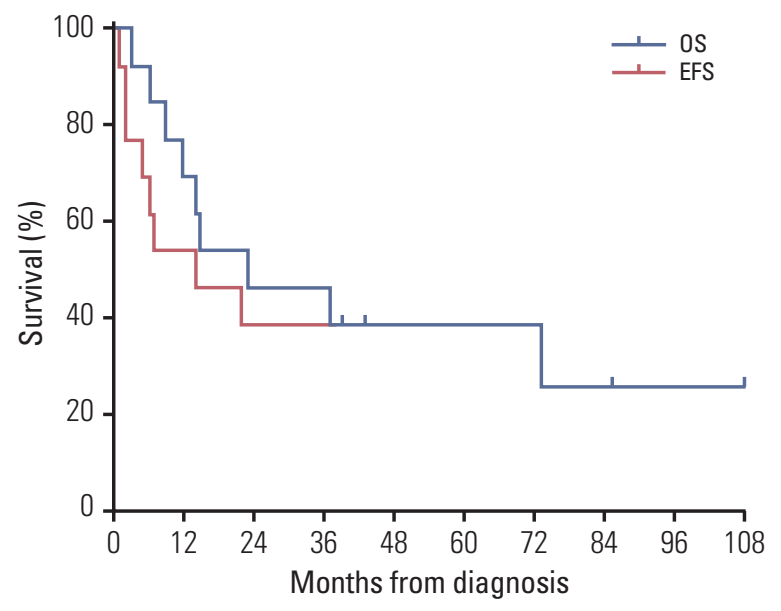

B

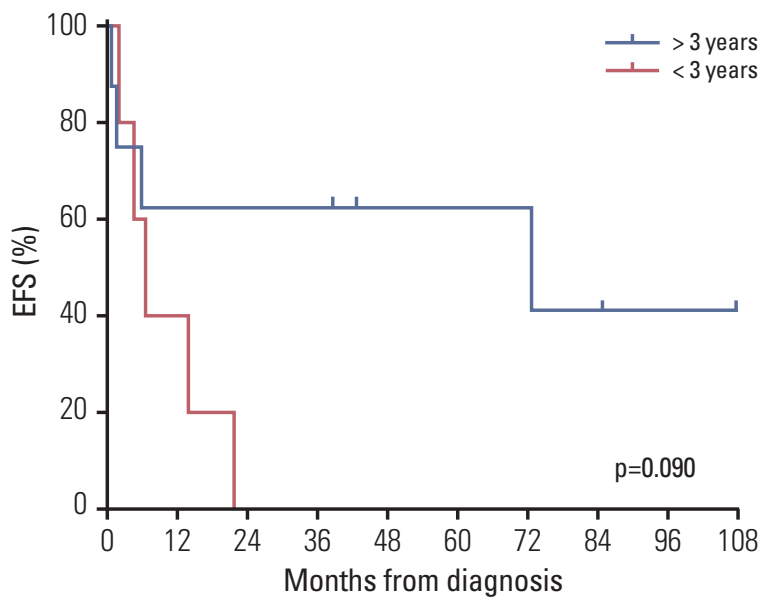

C

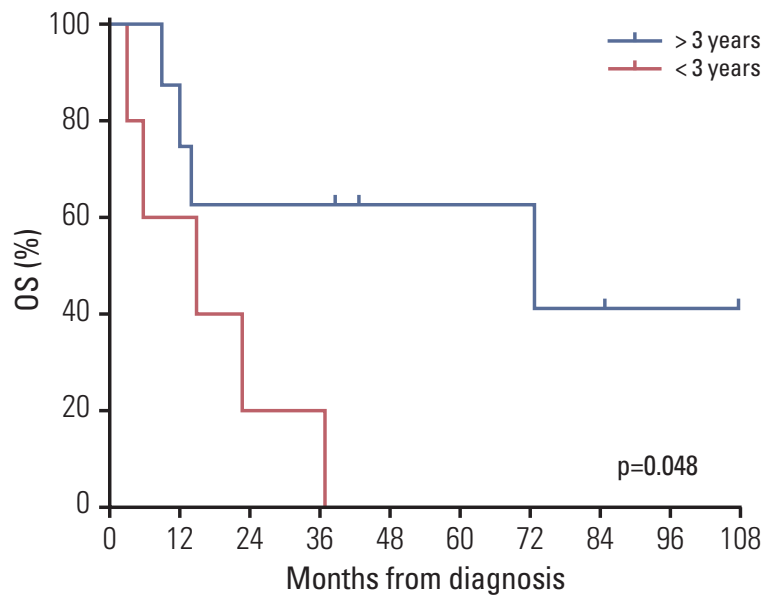

Fig. 2. Although all five young patients experienced relapse/progression and died from disease progression, four of the eight older patients remained event free at a median follow-up period of 64 months (range, 39 to 108 months) from diagnosis. For all patients, the 5-year event-free survival (EFS) and overall survival (OS) rates were $38.5 \pm 13.5 \%$ and $34.6 \pm 14.4 \%$, respectively.

young and one older) patients experienced relapse or progression after tandem HDCT/auto-SCT. The remaining four older patients remain progression free. Among eight patients who were in less than $\mathrm{CR}$ before tandem HDCT / auto-SCT, three patients achieved CR after tandem HDCT / auto-SCT and two patients remain event free. Two of five M0 older patients who received 23.4 Gy of CSRT experienced progression; however, the sites of progression were the primary sites. Although all five young patients experienced relapse/progression and died from disease progression, four of eight older patients remain progression free. Overall, nine patients experienced relapse/progression at a median follow-up period of 5 months (range, 1 to 73 months) from diagnosis and four patients remain event free at a median follow-up period of 64 months (range, 39 to 108 months) from diagnosis. For all patients, the 5-year EFS and OS rates were $38.5 \pm 13.5 \%$ and $34.6 \pm 14.4 \%$, respectively (Fig. 2). For young patients, the 5 -year EFS and OS rates were both zero. However, for older patients, the 5-year EFS and OS rates were $62.5 \pm 17.1 \%$ and $62.5 \pm 17.1 \%$, respectively.

\section{Late adverse effects}

Five patients who remained event free for more than 1 year 


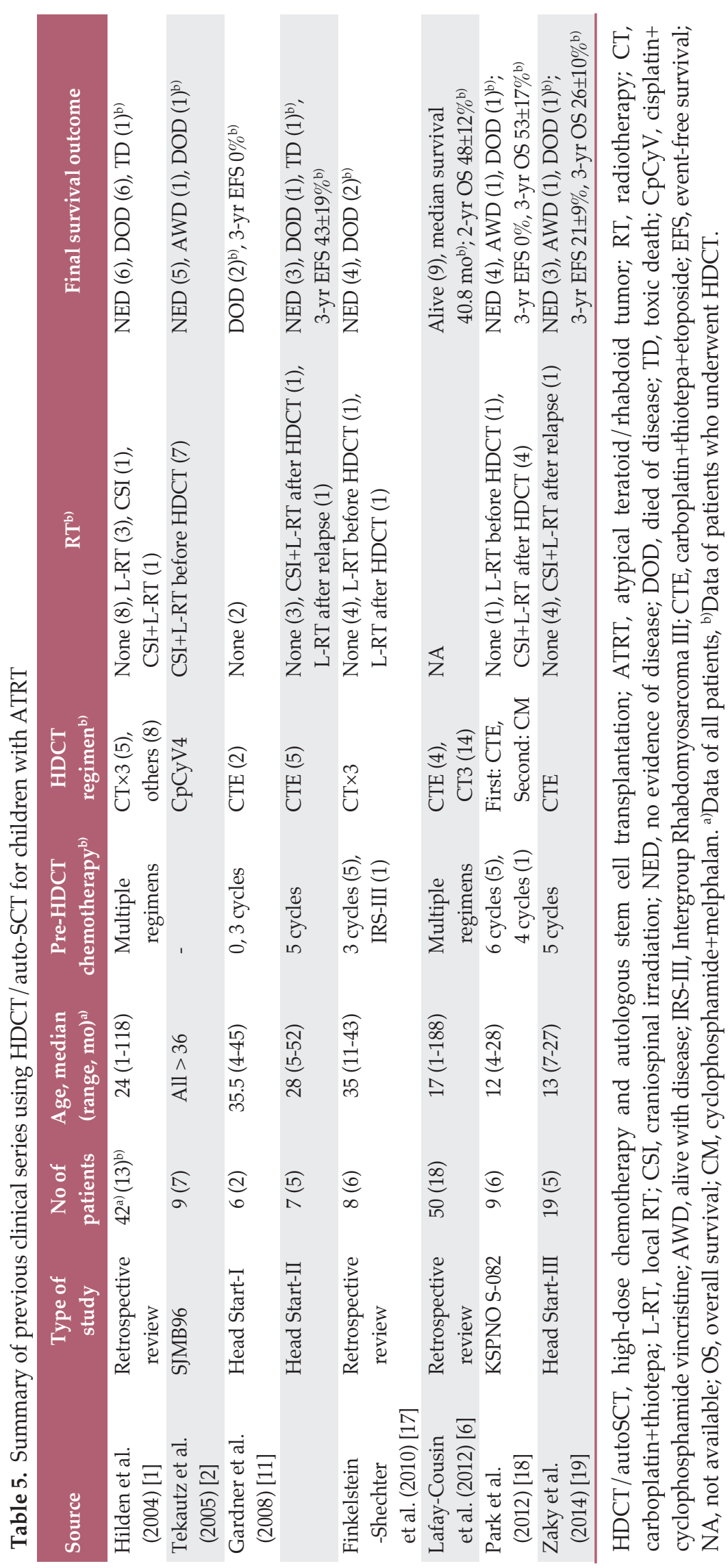


after HDCT/auto-SCT were evaluated for various late adverse effects. Late effects observed at a median of 35 months (range, 12 to 59 months) after the second HDCT/ auto-SCT were hypothyroidism $(n=1)$, growth hormone deficiency $(n=1)$, sex hormone deficiency $(n=2)$, and sensory neural hearing loss $(n=2)$. However, all late effects were grade $1 / 2$ toxicities. In two patients who received treatment during the prepubertal period, a deceleration in vertical growth was prominent and growth hormone replacement was initiated in one patient. The median value for full-scale IQ evaluated at a median of 21 months (range, 20 to 66 months) after RT was 80 (range, 55 to 103).

\section{Discussion}

The efficacy of many different treatments has been explored with the aim of improving the survival of ATRT patients; however, there is no consensus regarding standard chemotherapy. In the current study, CECV and CEIV regimens were used for induction treatment. Although toxicities were not significant with the exception of neutropenic fever, many patients experienced progression during induction chemotherapy. Gardner et al. [11] reported a possible benefit in patients who received methotrexate. In the study by Chi et al. [20] using the Intergroup Rhabdomyosarcoma III (IRSIII)-based regimen, the combination of intrathecal chemotherapy with focal radiation was an effective strategy for disease control in young patients. These findings suggest that incorporation of methotrexate, use of an IRS-III-based regimen, and inthrathecal chemotherapy might improve the outcome.

A few retrospective analyses and prospective studies have suggested that patients who received HDCT / auto-SCT have a higher survival rate than those who did not (Table 5). However, because of the small patient numbers, various chemotherapy regimens, and additional salvage treatment that often included RT, no real conclusions can be made from published data regarding the role of HDCT/auto-SCT in ATRT. In the current study we used tandem HDCT/autoSCT to further increase the intensity of chemotherapy. However, the number of patients enrolled was too small and patients were treated with several different modalities, therefore, it is still difficult to determine the role of tandem HDCT/auto-SCT. Our study has the same limitations of previous studies, as described above.

The optimal combination of regimens for tandem HDCT has not yet been determined. Variable intensity of tandem HDCT regimens and the length of the interval between the first and second HDCT/ auto-SCT cycles may affect outcome and toxicity profile. The intensities of our HDCT regimens are stronger or similar to those reported in previous studies for ATRT (Table 5). In our previous study, we showed that adequate rest is required between the first and second HDCT / auto-SCT cycles when using very intensive HDCT regimens [21]. In the current study, very intensive HDCT regimens were used in both the first and second HDCT/ auto-SCT cycles; therefore, a 12-week interval was implemented to reduce toxicities during the second HDCT / autoSCT. As a result, toxicities were manageable and there was no TRM during HDCT/auto-SCT in both young and older children. However, further dose-escalation during tandem HDCT/auto-SCT might be associated with more significant late adverse effects than single HDCT / auto-SCT. Therefore, longer follow-up and randomized trials including a larger cohort of patients are needed to determine whether the possible survival benefits of tandem HDCT/auto-SCT over single HDCT/auto-SCT ultimately outweigh the adverse effects associated with dose-intense tandem HDCT/autoSCT.

Unacceptable adverse effects of RT for young children with brain tumors led a number of institutions and national groups to adopt chemotherapy-based strategies designed to avoid or delay RT $[22,23]$. In the current study, we evaluated the effectiveness of tandem HDCT/ auto-SCT in young children with ATRT with the aim of both improving survival and avoiding or deferring RT until after the most radiosensitive neurodevelopmental mileposts. However, the outcome was very disappointing, mainly as a result of early progression. In our experience, intensive systemic chemotherapy alone was not an effective method to avoid or defer RT in young patients with ATRT, suggesting that RT should be considered much earlier in therapy. Some clinical trials now incorporate focal radiation at a much younger age than previously considered appropriate $[17,24,25]$. Intrathecal chemotherapy may also have potential benefit as an additional means to avoid RT or to intensify therapy in patients who are not candidates for CSRT $[5,20]$. Taken together, these data suggest that early administration of RT to the primary site together with intrathecal chemotherapy might prevent early progression and eventually improve the outcome, as reported in the study by Chi et al. [20].

In our recent study of patients older than 3 years with high-risk medulloblastoma, we were able to reduce the CSRT dose by employing tandem HDCT/ auto-SCT without jeopardizing survival rates [15]. The dose of CSRT was reduced to $23.4 \mathrm{~Gy}(\mathrm{M} 0)$ or $30.6 \mathrm{~Gy}(\mathrm{M}+)$ with the goal of reducing late adverse effects from CSRT. In that study, the probability of 5-year EFS was $70.0 \pm 10.3 \%$ for all patients and $70.6 \pm 11.1 \%$ for patients with metastasis. The same strategy was applied in the current study for ATRT patients older than 3 years. Three of five M0 patients who received 23.4 Gy of CSRT 
remained progression free and the site of progression in the two remaining patients was the primary tumor site, not metastatic sites. Findings of the current study suggest that 23.4 Gy of CSRT might be sufficient to prevent metastatic relapse in M0 patients older than 3 years if the intensity of chemotherapy is increased. However, further study is necessary.

While four of seven patients who could proceed to HDCT/auto-SCT without relapse or progression remain progression free after HDCT/auto-SCT, all three patients who underwent HDCT / auto-SCT as salvage treatment after relapse/progression experienced progression again. These findings suggest that pre-HDCT tumor status is important for prediction of outcome and careful consideration is required for selection of candidate patients for HDCT / autoSCT.

\section{Conclusion}

Intensive systemic chemotherapy including HDCT / autoSCT alone as a method to avoid RT is not effective in young children with disseminated ATRT. Therefore, early RT combined with or without intrathecal chemotherapy as a substitute for CSRT should be considered for young children. For older children without leptomeningeal seeding, our results suggest that dose-intense chemotherapy may decrease the required dose of CSRT without jeopardizing survival. However, further cooperative studies with a larger cohort of patients are needed to evaluate the effectiveness of our strategy.

\section{Conflicts of Interest}

Conflict of interest relevant to this article was not reported.

\section{Acknowledgments}

This study was supported by a grant from the National R\&D Program for Cancer Control, Ministry of Health and Welfare, Republic of Korea (No. 0520300).

\section{References}

1. Hilden JM, Meerbaum S, Burger P, Finlay J, Janss A, Scheithauer BW, et al. Central nervous system atypical teratoid/rhabdoid tumor: results of therapy in children enrolled in a registry. J Clin Oncol. 2004;22:2877-84.

2. Tekautz TM, Fuller CE, Blaney S, Fouladi M, Broniscer A, Merchant TE, et al. Atypical teratoid/rhabdoid tumors (ATRT): improved survival in children 3 years of age and older with radiation therapy and high-dose alkylator-based chemotherapy. J Clin Oncol. 2005;23:1491-9.

3. Bikowska B, Grajkowska W, Jozwiak J. Atypical teratoid/ rhabdoid tumor: short clinical description and insight into possible mechanism of the disease. Eur J Neurol. 2011;18: 813-8.

4. von Hoff K, Hinkes B, Dannenmann-Stern E, von Bueren AO, Warmuth-Metz M, Soerensen N, et al. Frequency, riskfactors and survival of children with atypical teratoid rhabdoid tumors (AT / RT) of the CNS diagnosed between 1988 and 2004, and registered to the German HIT database. Pediatr Blood Cancer. 2011;57:978-85.

5. Athale UH, Duckworth J, Odame I, Barr R. Childhood atypical teratoid rhabdoid tumor of the central nervous system: a meta-analysis of observational studies. J Pediatr
Hematol Oncol. 2009;31:651-63.

6. Lafay-Cousin L, Hawkins C, Carret AS, Johnston D, Zelcer S, Wilson B, et al. Central nervous system atypical teratoid rhabdoid tumours: the Canadian Paediatric Brain Tumour Consortium experience. Eur J Cancer. 2012;48:353-9.

7. Mulhern RK, Merchant TE, Gajjar A, Reddick WE, Kun LE. Late neurocognitive sequelae in survivors of brain tumours in childhood. Lancet Oncol. 2004;5:399-408.

8. Matthay KK, Reynolds CP, Seeger RC, Shimada H, Adkins ES, Haas-Kogan D, et al. Long-term results for children with high-risk neuroblastoma treated on a randomized trial of myeloablative therapy followed by 13-cis-retinoic acid: a children's oncology group study. J Clin Oncol. 2009;27:1007-13.

9. Fangusaro J, Finlay J, Sposto R, Ji L, Saly M, Zacharoulis S, et al. Intensive chemotherapy followed by consolidative myeloablative chemotherapy with autologous hematopoietic cell rescue (AuHCR) in young children with newly diagnosed supratentorial primitive neuroectodermal tumors (sPNETs): report of the Head Start I and II experience. Pediatr Blood Cancer. 2008;50:312-8.

10. Mason WP, Grovas A, Halpern S, Dunkel IJ, Garvin J, Heller 
$\mathrm{G}$, et al. Intensive chemotherapy and bone marrow rescue for young children with newly diagnosed malignant brain tumors. J Clin Oncol. 1998;16:210-21.

11. Gardner SL, Asgharzadeh S, Green A, Horn B, McCowage G, Finlay J. Intensive induction chemotherapy followed by high dose chemotherapy with autologous hematopoietic progenitor cell rescue in young children newly diagnosed with central nervous system atypical teratoid rhabdoid tumors. Pediatr Blood Cancer. 2008;51:235-40.

12. Sung KW, Yoo KH, Cho EJ, Koo HH, Lim DH, Shin HJ, et al. High-dose chemotherapy and autologous stem cell rescue in children with newly diagnosed high-risk or relapsed medulloblastoma or supratentorial primitive neuroectodermal tumor. Pediatr Blood Cancer. 2007;48:408-15.

13. Gajjar A, Chintagumpala M, Ashley D, Kellie S, Kun LE, Merchant TE, et al. Risk-adapted craniospinal radiotherapy followed by high-dose chemotherapy and stem-cell rescue in children with newly diagnosed medulloblastoma (St Jude Medulloblastoma-96): long-term results from a prospective, multicentre trial. Lancet Oncol. 2006;7:813-20.

14. Sung KW, Lim DH, Lee SH, Yoo KH, Koo HH, Kim JH, et al. Tandem high-dose chemotherapy and auto-SCT for malignant brain tumors in children under 3 years of age. Bone Marrow Transplant. 2013;48:932-8.

15. Sung KW, Lim DH, Son MH, Lee SH, Yoo KH, Koo HH, et al. Reduced-dose craniospinal radiotherapy followed by tandem high-dose chemotherapy and autologous stem cell transplantation in patients with high-risk medulloblastoma. Neuro Oncol. 2013;15:352-9.

16. Laurent JP, Chang CH, Cohen ME. A classification system for primitive neuroectodermal tumors (medulloblastoma) of the posterior fossa. Cancer. 1985;56:1807-9.

17. Finkelstein-Shechter T, Gassas A, Mabbott D, Huang A, Bartels U, Tabori U, et al. Atypical teratoid or rhabdoid tumors: improved outcome with high-dose chemotherapy. J Pediatr Hematol Oncol. 2010;32:e182-6.

18. Park ES, Sung KW, Baek HJ, Park KD, Park HJ, Won SC, et al.
Tandem high-dose chemotherapy and autologous stem cell transplantation in young children with atypical teratoid/rhabdoid tumor of the central nervous system. J Korean Med Sci. 2012;27:135-40.

19. Zaky W, Dhall G, Ji L, Haley K, Allen J, Atlas M, et al. Intensive induction chemotherapy followed by myeloablative chemotherapy with autologous hematopoietic progenitor cell rescue for young children newly-diagnosed with central nervous system atypical teratoid/ rhabdoid tumors: the Head Start III experience. Pediatr Blood Cancer. 2014;61:95-101.

20. Chi SN, Zimmerman MA, Yao X, Cohen KJ, Burger P, Biegel JA, et al. Intensive multimodality treatment for children with newly diagnosed CNS atypical teratoid rhabdoid tumor. J Clin Oncol. 2009;27:385-9.

21. Lee SH, Son MH, Sung KW, Choi YB, Lee NH, Yoo KH, et al. Toxicity of tandem high-dose chemotherapy and autologous stem cell transplantation using carboplatin-thiotepa-etoposide and cyclophosphamide-melphalan regimens for malignant brain tumors in children and young adults. J Neurooncol. 2014;120:507-13.

22. Duffner PK, Horowitz ME, Krischer JP, Friedman HS, Burger PC, Cohen ME, et al. Postoperative chemotherapy and delayed radiation in children less than three years of age with malignant brain tumors. N Engl J Med. 1993;328:1725-31.

23. Grill J, Sainte-Rose C, Jouvet A, Gentet JC, Lejars O, Frappaz $\mathrm{D}$, et al. Treatment of medulloblastoma with postoperative chemotherapy alone: an SFOP prospective trial in young children. Lancet Oncol. 2005;6:573-80.

24. Pai Panandiker AS, Merchant TE, Beltran C, Wu S, Sharma S, Boop FA, et al. Sequencing of local therapy affects the pattern of treatment failure and survival in children with atypical teratoid rhabdoid tumors of the central nervous system. Int J Radiat Oncol Biol Phys. 2012;82:1756-63.

25. Chen YW, Wong TT, Ho DM, Huang PI, Chang KP, Shiau CY, et al. Impact of radiotherapy for pediatric CNS atypical teratoid / rhabdoid tumor (single institute experience). Int J Radiat Oncol Biol Phys. 2006;64:1038-43. 